\title{
Bringing Home The Bacon: Update on The State of Kidney Xenotransplantation
}

\author{
David K.C. Cooper ${ }^{a} \quad$ H. Iwase ${ }^{a} \quad$ L. Wang $^{a, b} \quad$ T. Yamamoto $^{\mathrm{a}} \quad$ Qi Li ${ }^{\mathrm{a}, \mathrm{b}} \quad$ J. Li ${ }^{\mathrm{a}, \mathrm{b}}$ \\ H. Zhou a, C H. Hara ${ }^{\text {a }}$ \\ ${ }^{a}$ Xenotransplantation Program, Department of Surgery, University of Alabama at Birmingham, Birmingham, $A L$, \\ USA; 'becond Affiliated Hospital, University of South China, Hengyang, China; ' Huazhong University of Science and \\ Technology, Tongji Hospital, Wuhan, China
}

\section{Keywords}

End-stage renal disease $\cdot$ Kidney $\cdot$ Pig geneticallyengineered $\cdot$ Xenotransplantation

\begin{abstract}
Background: There is a continuing critical shortage of organs from deceased human donors for transplantation, particularly for patients awaiting kidney transplantation. Efforts are being made to resolve the donor kidney shortage by the transplantation of kidneys from genetically-engineered pigs. Summary: This review outlines the pathobiological barriers to pig organ xenotransplantation in primates, which include (i) antibody-dependent complement-mediated rejection, (ii) a T cell-mediated elicited antibody and cellular response, (iii) coagulation dysregulation between pigs and primates, and (iv) a persistent inflammatory response. As a result of increasing genetic manipulation of the pig and the introduction of novel immunosuppressive agents, pig kidney graft survival has increased from minutes to months, and even to $>1$ year in some cases. Aspects of the selection of the patients for a first clinical trial are discussed. Although there would appear to be some cross-reactivity between an-
\end{abstract}

๑ 2018 S. Karger AG, Basel ti-human leukocyte antigen (HLA) antibodies and swine leukocyte antigens expressed in pigs, some HLA-sensitized patients will be at no disadvantage if they receive a pig kidney. Furthermore, the current limited evidence is that, even if the patient becomes sensitized to pig antigens (after a pig organ transplant), this would not be detrimental to a subsequent allotransplant. The potential risk of infection with a pig microorganism, and the function of a pig kidney in a primate are also discussed. Key Message: The recent encouraging results of pig kidney transplantation in nonhuman primates suggest the likelihood of a successful (and safe) initial clinical trial, with graft survival for months or possibly years.

(c) 2018 S. Karger AG, Basel

\section{Introduction}

There is a continuing shortage of organs from deceased human donors for transplantation, especially for patients awaiting kidney transplantation, despite the ability of some patients to provide a living donor. Efforts are being made to resolve the donor shortage by the transplantation of kidneys from genetically-engineered pigs. 
Table 1. The advantages and disadvantages of the pig as a potential source of organs and cells for humans, in contrast with those of the baboon in this role

\begin{tabular}{lll}
\hline & Pig & Baboon \\
\hline Availability & Unlimited & Limited \\
Breeding potential & Good & Poor \\
Period to reproductive maturity & $4-8$ months & $3-5$ years \\
Length of pregnancy & $114+2$ days & $173-193$ days \\
Number of offspring & $5-12$ & $1-2$ \\
Growth & Rapid & Slow \\
& (adult human size within 6 months)** & (9 years to reach maximum size) \\
Size of adult organs & Adequate & Inadequate* \\
Cost of maintenance & Significantly lower & High \\
Anatomical similarity to humans & Moderately close & Close \\
Physiological similarity to humans & Moderately close & Close \\
Relationship of immune system to humans & Distant & Limited \\
Knowledge of tissue typing & Considerable (in selected herds) & Important \\
Necessity for blood type compatibility with humans & Probably unimportant & None \\
Experience with genetic engineering & Considerable & High \\
Risk of transfer of infection (xenozoonosis) & Low & No \\
Availability of designated pathogen-free animals & Yes & More in favor \\
Public opinion & Mixed \\
\hline
\end{tabular}

* The size of certain organs, for example, the heart, would be inadequate for transplantation into adult humans.

** Breeds of miniature swine are approximately $50 \%$ of the weight of domestic pigs at birth and sexual maturity, and reach a maximum weight of approximately $30 \%$ of standard breeds.

Xenotransplantation has a long clinical history, with the first animal kidney transplant being carried out more than a century ago [1].

\section{Advantages of the Pig as a Source of Organs for Clinical Transplantation}

Although nonhuman primates (NHPs) are closer to humans from an immunological perspective, this is counteracted by several advantages of the pig (Table 1). Nevertheless, it has required 3 decades of intensive research for the pathobiological barriers to pig xenotransplantation to be identified and overcome.

\section{Pathobiological Barriers}

Four major barriers have been identified [2].

\section{Antibody-Dependent Complement-Mediated \\ Rejection}

Believed to be as a defense mechanism against various viruses and bacteria that colonize their gastrointestinal tracts during infancy, humans develop "natural" antibodies to certain carbohydrate (glycan) antigens that are expressed on these microorganisms [3]. As pig vascular endothelial and certain other cells express these same glycans, when a pig organ is transplanted into a human or NHP recipient, these preformed antibodies immediately bind to the graft, activating the complement cascade, and destroying it, usually within minutes ("hyperacute" rejection).

Steps to overcome this major barrier became possible after the most important glycan target for anti-pig antibodies was identified by Good et al. [4]. The glycan was identified as galactose- $\alpha 1,3$-galactose (Gal). Methods of plasmapheresis and immunoadsorption to remove the anti-pig antibodies from the plasma of the potential recipient proved only partially successful as the antibodies returned rapidly, causing delayed antibody-mediated rejection. Although suggested in 1993, it was not until genetic-engineering enabled deletion of expression of Gal in pigs [5].

The production of pigs that did not express Gal ( $\alpha 1,3-$ galactosyltransferase gene-knockout pigs) in 2003 was a major step forward [6]. Organs from these pigs, transplanted into NHPs, were no longer susceptible to hyper- 
acute rejection, though a delayed, reduced antibody response to other (at that time, unknown) pig antigens, but termed "nonGal" antigens, remained problematic.

In the meantime, a second approach to overcoming hyperacute rejection had been proven efficacious, namely the transgenic expression of a human complement-regulatory protein in the pig [7]. When activated, human complement rarely injures the patient's own tissues. Pigs have their own pig complement-regulatory proteins, but these are not very efficient at protecting against human complement. Organs from pigs expressing one or more human complement-regulatory proteins, for example, CD55 (decay accelerating factor), CD46, or CD59, are largely protected from hyperacute rejection, but remain susceptible to delayed antibody-mediated rejection even when the NHP recipient is administered intensive pharmacologic immunosuppressive therapy.

Organs from $\alpha 1,3$-galactosyltransferase gene-knockout pigs that also express a human complement-regulatory protein were proven to be even more protected than pigs with either genetic modification alone [8].

Two nonGal antigens have subsequently been identified, namely $\mathrm{N}$-glycolylneuraminic acid [9] and Sda $(\beta 4 \mathrm{GalNT} 2)$ [10]. When expression of one or both of these has been deleted from the organ-source pig by knockout technology, antibody binding has been reduced further $[11,12]$.

\section{T Cell-Mediated Elicited Antibody and Cellular \\ Response}

Although $\mathrm{T}$ cell infiltration was not obvious in pig grafts (largely because the antibody-mediated response was overwhelming before cellular infiltration of the graft had taken place), it became clear that, unless effective pharmacologic immunosuppressive therapy was administered to the recipient, rejection would develop through the development of a de novo T cell-mediated elicited antibody response. Conventional agents, for example, cyclosporine, tacrolimus (that block signal 1 of the $\mathrm{T}$ cell immune response), proved less than totally effective unless administered at very high doses, but the new costimulation blockade agents, for example, anti-CD154mAb, anti-CD40mAb (that block signal 2), successfully prevented a $T$ cell response [13]. Today, almost 20 years later, with pigs that express neither Gal nor the 2 nonGal antigens, conventional immunosuppressive therapy is proving more effective [Iwase et al., unpubl.].

The $\mathrm{T}$ cell response may also be reduced by genetic engineering of the pig, for example, by the introduction of a mutant gene that reduces MHC class II (swine leuko- cyte antigen [SLA] class II) expression [14], or by knockout of MHC class I (SLA class I) [15]. The transgenic expression of a co-stimulation blockade agent, for example, CTLA4-Ig, to provide local suppression of the T cell response, has also proved possible [6].

Coagulation Dysregulation between Pigs and Primates

Even a low level of anti-nonGal antibody in the recipient was found to be associated with loss of the graft over days, weeks, or months by the development of thrombotic microangiopathy in the graft and/or consumptive coagulopathy in the recipient [16]. This was believed to be related to activation of the vascular endothelium, converting its usual anticoagulant state into a procoagulant state, which was in part associated with molecular incompatibilities between the pig and primate coagulation-anticoagulation systems [17]. These complications could be largely prevented by expression in the organ-source pig of one or more human coagulation-regulatory proteins, for example, thrombomodulin or endothelial protein $\mathrm{C}$ receptor. Prolonged graft survival then became possible, with heterotopic (non-lifesupporting) heart grafts functioning for $>2$ years and lifesupporting kidney grafts for many months [18-20].

\section{Inflammatory Response}

A prolonged and persistent inflammatory response to even a small pig xenograft, for example, an artery patch, was demonstrated to precede the coagulation disturbance [21]. As inflammation can augment an immune response, the inflammatory response has been suppressed by the administration of agents such as the interleukin- 6 receptor blocker, tocilizumab, and the anti-tumor necrosis factor-alpha agent, etanercept.

It is possible to introduce an "anti-inflammatory" transgene, for example, A20, hemeoxygenase-1, into pigs, but whether this will be sufficient to protect the graft from a systemic inflammatory response remains uncertain.

\section{Current Status of Pig Kidney Transplantation in NHPs}

As a result of the increasing genetic manipulation of the pig, with some pigs now expressing six different genetic modifications, the results of pig organ transplantation in immunosuppressed NHPs have improved considerably. Maximum pig kidney graft survival has increased from minutes to months, and occasionally to $>1$ year (Fig. 1) [19, 20, 22, 23]. These encouraging results have led to consideration of initial clinical trials. 


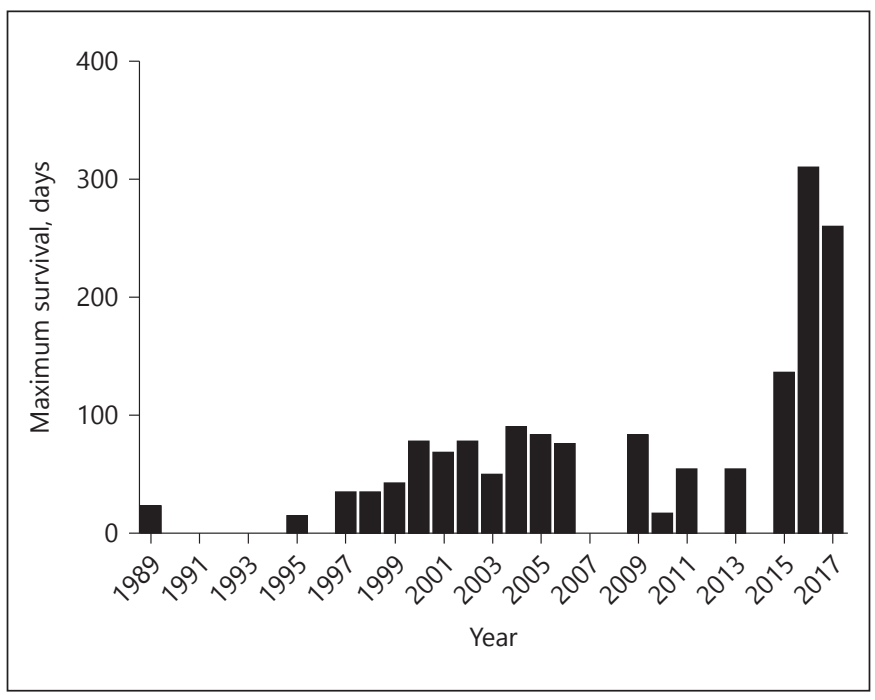

Fig. 1. Maximal pig kidney graft survival in a nonhuman primate by year. Maximum survival has increased from 22 days in 1989, to 90 days in 2004, and to >300 days in 2016 .

\section{Initial Clinical Trials of Xenotransplantation}

Trials of encapsulated wild-type (genetically-unmodified) pig pancreatic islets in patients with diabetes have been carried out, with proven safety but with only a modest impact on the control of glycemia. Clinical trials of decellularized pig corneal transplantation are being undertaken in China, but no recent trial of solid organ transplantation has been initiated.

The case for an initial trial of "free" (non-encapsulated) islet transplantation in immunosuppressed patients is strong because, if the islets are rejected, there is little risk to the patient who can return to insulin therapy. However, there is some resistance to committing a patient with diabetes to life-long immunosuppressive therapy.

In patients presenting with fulminant hepatic failure, bridging with an auxiliary (heterotopic) pig liver transplant may be a feasible option until either (i) the patient's own liver recovers, or (ii) human liver allotransplantation can be carried out. However, because of additional coagulation dysfunction, the current results of pig liver xenotransplantation in NHPs are poor in comparison with those of heart and kidney transplantation, with survival extending to approximately one month [24]. Further genetic modification of the pig is required. Similarly, transplantation of the pig lung is proving much more difficult than of other organs [25], and so cannot yet be considered for a clinical trial.

Kidney Xenotransplantation
Patients with severe heart failure awaiting heart transplantation can be supported for months or years by the currently-available ventricular assist devices, and therefore the ethics of committing them to xenotransplantation have to be carefully considered. However, in neonates, infants, and small children with complex congenital heart defects, such as hypoplastic left heart syndrome, no suitable device is available. As the results of palliative surgery are less than optimal, and there is a significant mortality while the patient waits for a deceased human donor heart to become available, xenotransplantation may be indicated.

Although dialysis is available to patients with endstage renal disease, the waiting time to be allocated a donor kidney for many (particularly older patients, for example, $>60$ years of age) is so long that they may not live long enough to benefit from allotransplantation. For some of them, a pig xenograft might prove a welcome alternative to the restricted life associated with chronic dialysis. Additional potential patients include those with severely limited vascular access, and those with rapidlyrecurring renal disease [26]. It is unknown whether these diseases will recur in a pig graft, but repeat allotransplantation is often not indicated, and a deceased human donor kidney can be allocated to a more suitable recipient in whom the graft will function for significantly longer.

Furthermore, although there would appear to be some cross-reactivity between anti-human leukocyte antigen (HLA) antibodies and SLA, some patients with a high level of panel-reactive antibodies, whose wait for a compatible deceased human donor may be even longer, would appear to be able to receive a pig kidney graft without risk of hyperacute or early antibody-mediated rejection. In addition, the current limited evidence is that, even if the patient becomes sensitized to pig antigens (after a pig organ transplant), this would not be detrimental to a subsequent allotransplant.

\section{Other Aspects of Xenotransplantation}

\section{The Potential Risk of Infection with a Pig}

\section{Microorganism}

There are significant risks associated with allotransplantation. For example, transmission of viruses, such as cytomegalovirus, Epstein-Barr virus, and/or BK virus, is common, and can be associated with complications, but is accepted knowingly as few donor organs would otherwise become available. Occasionally, a more serious microorganism, for example, a hepatitis virus, HIV, rabies 
(or a malignant tumor), is unknowingly transferred with the organ with fatal or serious outcome.

In this respect, the pigs that will provide organs for clinical xenotransplantation will be much safer. As sources of a biological "product," they will be held to a much higher safety standard than is a human donor [27]. They will be bred and housed under clean, biosecure conditions, and sentinel animals will be tested at intervals to ensure an absence of all known microorganisms considered detrimental if transferred with the organ. However, there remain potential risks associated with the transfer of (i) porcine endogenous retroviruses (PERVs) or (ii) unknown viruses. The risks of a clinical infection from a PERV are today considered to be low and, in any case, could likely be treated with currently-available drugs. Although inactivation of PERVs is now possible, it is not considered to be either essential or totally effective. Although there will always be a risk of a hitherto "unknown" infection, this should not prevent advancing to the clinic if the benefits to patients are likely to be considerable. However, life-long monitoring of the patient will be required.

\section{Physiological Aspects of Xenotransplantation}

The current evidence is that a pig kidney will function well in a human recipient, as they have in NHPs [20]. When the immune response is controlled, there has been no evidence of significant proteinuria, and electrolytes have been maintained within normal ranges. It is not yet certain whether pig erythropoietin is functional in primates but, if not, human recombinant erythropoietin can be administered or the organ-source pig can be rendered transgenic for human erythropoietin. A rapid increase in size of the pig kidney graft has been documented during the first few weeks in some (but not all) NHP studies [20, 28], and it is uncertain whether this is related to (i) the transplantation of a single immature kidney into a more mature NHP, or (ii) retained pig growth hormone in the kidney. It is known that human growth hormone is active in pigs. After the first 1-3 months, growth has been reduced to that of a native NHP kidney.

\section{Conclusions}

The recent encouraging results of pig kidney transplantation in NHPs suggest the likelihood of a successful (and safe) initial clinical trial, with graft survival for months or years. However, at present, the costimulation blockade agents that have been administered in experi- mental models are not yet approved for clinical use in the USA, and so it is important to demonstrate that rejection can be prevented by FDA-approved pharmacologic or biologic agents. The potential risk of a pig microorganism being transferred to the patient is considered small. The timing of such a clinical trial will to some extent depend on the requirements of the regulatory authorities [29].

The ultimate goal of both allotransplantation and xenotransplantation is to achieve a state of immunological tolerance in which, even in the absence of exogenous immunosuppressive therapy, the recipient no longer attempts to reject the graft. This has been achieved in a very small number of patients with kidney allotransplants through the induction of hematopoietic cell transplantation. This approach, and tolerance through thymus transplantation, are being explored in xenotransplantation models. The ability to genetically-engineer the pig may make tolerance easier to achieve than in allotransplantation.

\section{Acknowledgements}

Work on xenotransplantation at the University of Alabama at Birmingham is supported in part by NIH NIAID U19 grant AI090959.

\section{Disclosure Statement}

No author declares a conflict of interest.

References

1 Cooper DK. A brief history of cross-species organ transplantation. Proc (Bayl Univ Med Cent) 2012;25:49-57.

2 Cooper DK, Ezzelarab MB, Hara H, Iwase H, Lee W, Wijkstrom M, et al: The pathobiology of pig-to-primate xenotransplantation: a historical review. Xenotransplantation 2016;23: 83-105.

3 Galili U, Mandrell RE, Hamadeh RM, Shohet SB, Griffiss JM: Interaction between human natural anti-alpha-galactosyl immunoglobulin $\mathrm{G}$ and bacteria of the human flora. Infect Immun 1988;56:1730-1737.

4 Good AH, Cooper DK, Malcolm AJ, Ippolito RM, Koren E, Neethling FA, et al: Identification of carbohydrate structures that bind human antiporcine antibodies: implications for discordant xenografting in humans. Transplant Proc 1992;24:559-562.

5 Phelps CJ, Koike C, Vaught TD, Boone J, Wells KD, Chen SH, et al: Production of alpha 1,3-galactosyltransferase-deficient pigs. Science 2003;299:411-414. 
6 Cooper DK, Ekser B, Ramsoondar J, Phelps C, Ayares D: The role of genetically engineered pigs in xenotransplantation research. J Pathol 2016;238:288-299.

7 Cozzi E, White DJ: The generation of transgenic pigs as potential organ donors for humans. Nat Med 1995;1:964-966.

8 Azimzadeh AM, Kelishadi SS, Ezzelarab MB, Singh AK, Stoddard T, Iwase H, et al: Early graft failure of GalTKO pig organs in baboons is reduced by expression of a human complement pathway-regulatory protein. Xenotransplantation 2015;22:310-316.

9 Padler-Karavani V, Varki A: Potential impact of the non-human sialic acid N-glycolylneuraminic acid on transplant rejection risk. Xenotransplantation 2011;18:1-5.

10 Byrne GW, Du Z, Stalboerger P, Kogelberg $\mathrm{H}, \mathrm{McGregor} \mathrm{CG}$ : Cloning and expression of porcine beta1,4 $\mathrm{N}$-acetylgalactosaminyl transferase encoding a new xenoreactive antigen. Xenotransplantation 2014;21:543-554.

11 Lutz AJ, Li P, Estrada JL, Sidner RA, Chihara RK, Downey SM, et al: Double knockout pigs deficient in $\mathrm{N}$-glycolylneuraminic acid and galactose alpha-1,3-galactose reduce the humoral barrier to xenotransplantation. Xenotransplantation 2013;20:27-35.

12 Estrada JL, Martens G, Li P, Adams A, Newell KA, Ford ML, et al: Evaluation of human and non-human primate antibody binding to pig cells lacking GGTA1/CMAH/ß4GalNT2 genes. Xenotransplantation 2015;22:194202.

13 Buhler L, Awwad M, Basker M, Gojo S, Watts A, Treter S, et al: High-dose porcine hematopoietic cell transplantation combined with CD40 ligand blockade in baboons prevents an induced anti-pig humoral response. Transplantation 2000;69:2296-2304.

14 Hara H, Witt W, Crossley T, Long C, Isse K, Fan L, et al: Human dominant-negative class
II transactivator transgenic pigs - effect on the human anti-pig $\mathrm{T}$-cell immune response and immune status. Immunology 2013;140: 39-46.

15 Reyes LM, Estrada JL, Wang ZY, Blosser RJ, Smith RF, Sidner RA, et al: Creating class I MHC-null pigs using guide RNA and the Cas9 endonuclease. J Immunol 2014;193: 5751-5757.

16 Buhler L, Basker M, Alwayn IP, Goepfert C, Kitamura H, Kawai T, et al: Coagulation and thrombotic disorders associated with pig organ and hematopoietic cell transplantation in nonhuman primates. Transplantation 2000; 70:1323-1331.

17 Cowan PJ, Robson SC, d'Apice AJ. Controlling coagulation dysregulation in xenotransplantation. Curr Opin Organ Transplant 2011;16:214-221.

18 Mohiuddin MM, Singh AK, Corcoran PC, Thomas ML 3rd, Clark T, Lewis BG, et al: Chimeric 2C10R4 anti-CD40 antibody therapy is critical for long-term survival of GTKO. hCD46.hTBM pig-to-primate cardiac xenograft. Nat Commun 2016;7:11138.

19 Iwase H, Liu H, Wijkstrom M, Zhou H, Singh J, Hara $\mathrm{H}$, et al: Pig kidney graft survival in a baboon for 136 days: longest life-supporting organ graft survival to date. Xenotransplantation 2015;22:302-309.

20 Iwase H, Hara H, Ezzelarab M, Li T, Zhang Z, Gao B, et al: Immunological and physiological observations in baboons with life-supporting genetically engineered pig kidney grafts. Xenotransplantation 2017;24.

21 Ezzelarab MB, Ekser B, Azimzadeh A, Lin CC, Zhao Y, Rodriguez R, et al: Systemic inflammation in xenograft recipients precedes activation of coagulation. Xenotransplantation 2015;22:32-47.

22 Higginbotham L, Mathews D, Breeden CA, Song M, Farris AB 3rd, Larsen CP, et al: Pre- transplant antibody screening and antiCD154 costimulation blockade promote long-term xenograft survival in a pig-to-primate kidney transplant model. Xenotransplantation 2015;22:221-230.

23 Higginbotham L, Kim S, Mathews D, Stephenson A, Breeden C, Larsen C, Ford M, Newell K, Tector A, Adams A: Late renal xenograft failure is antibody-mediated: description of the longest-reported survival in pig-toprimate renal xenotransplantation. Am Transplant Congress 2016;Abstract \#A6.

24 Shah JA, Patel MS, Elias N, Navarro-Alvarez N, Rosales I, Wilkinson RA, et al: Prolonged survival following pig-to-primate liver xenotransplantation utilizing exogenous coagulation factors and costimulation blockade. Am J Transplant 2017;17:2178-2185.

25 Laird C, Burdorf L, Pierson RN 3rd: Lung xenotransplantation: a review. Curr Opin Organ Transplant 2016;21:272-278.

26 Cooper DKC, Wijkstrom M, Hariharan S, Chan JL, Singh A, Horvath K, et al: Selection of patients for initial clinical trials of solid organ xenotransplantation. Transplantation 2017;101:1551-1558.

27 Onions D, Cooper DK, Alexander TJ, Brown C, Claassen E, Foweraker JE, et al: An approach to the control of disease transmission in pig-to-human xenotransplantation. Xenotransplantation 2000;7:143-155.

28 Tanabe T, Watanabe H, Shah JA, Sahara H, Shimizu A, Nomura S, et al: Role of intrinsic (graft) versus extrinsic (host) factors in the growth of transplanted organs following allogeneic and xenogeneic transplantation. Am J Transplant 2017;17:1778-1790.

29 Cooper DKC, Pierson RN 3rd, Hering BJ, Mohiuddin MM, Fishman JA, Denner J, et al: Regulation of clinical xenotransplantationtime for a reappraisal. Transplantation 2017; 101:1766-1769. 